\title{
Influence of Skyrme-type interaction on HICs observables
}

\author{
YingXun Zhang ${ }^{1}$, M.B.Tsang ${ }^{2}$, Zhuxia Li $^{1}$ and HAng LiU ${ }^{3}$
}

1 China Institute of Atomic Energy, Beijing 102413, P.R.China

2 National Superconducting Cyclotron Laboratory, Michigan State University, East Lansing, MI 48824, USA

3 Texas Advanced Computing Center, University of Texas, Austin, TX 78758, USA

\begin{abstract}
A new version of the improved quantum molecular dynamics model has been developed by including Skyrme type momentum dependent interaction. 12 Skyrme like parameter sets $\left\{K_{0}, S_{0}, L, m_{s}^{*}, m_{v}^{*}\right\}$ are adopted in the transport model code to calculate the isospin diffusion, single and double ratios of transverse emitted nucleons, neutron proton isoscaling ratios. The calculations and correlation analysis evidence that isospin diffusion observable at lower beam energy is sensitive to the slope of symmetry energy and $m_{s}^{*}$. The high energy neutrons and protons yield ratios from reactions at different incident energies provide a sensitive observable to study the nucleon effective mass splitting, at higher beam energy.
\end{abstract}

The properties of isospin asymmetric nuclear equation of state is of fundamental importance in our understanding of nature's asymmetric objects including neutron stars as well as heavy nuclei with very different number of neutrons and protons. Currently, there exist, lot of effective interaction parameter sets $[1,2]$, for fitting the ground-state properties of stable nuclei, symmetric and asymmetric nuclear matter. It also leads very different neutron Equation of state which may have different nuclear matter (NM) parameters, such as incompressibility $K_{0}$, isoscalar effective mass $m_{s}^{*}$, symmetry energy coefficient $S_{0}$, slope of symmetry energy $L$ and isovector effec- 
tive mass $m_{v}^{*}[3,4]$, or different sign or magnitude of effective mass splitting. Thus, constraining isospin asymmetric nuclear equation of state become one of hot topics in nuclear physics, especially for heavy ion collisions.

Some properties of symmetric nuclear matter, such as $K_{0}=230 \pm 30 \mathrm{MeV}$ $[1,5,6]$ and $m_{s}^{*} / m=0.65-0.99[1,5,7,8]$, have been extracted from isoscalar collective vibrations, giant quadrupole resonance and heavy ion collisions measurements. Recently, a consistent set of constraints on the symmetry energy near saturation density, i.e. $S_{0} \sim 31 \mathrm{MeV} \pm \Delta S_{0}$ and its slope $L \sim$ $50-60 \mathrm{MeV} \pm \Delta L$, has been obtained from observables measured in both nuclear structure and nuclear reaction experiments [9-12]. Up to now, the challenge of symmetry energy constraints around normal density is how to reduce the uncertainties of the constraints on symmetry energy, i.e. how to reduce $\Delta L$ and $\Delta S_{0}$ or obtain precise and accurate symmetry energy constraints. As a first step to reach this goal, one has to quantitatively understand where the uncertainties of symmetry energy come from, and then to identify what experimental observables are crucial for better constraining the interested physical quantities in the theoretical models [13]. In this work, we firstly investigate the correlation between symmetry energy and effective mass, effective mass splitting within the framework of Skyrme-Hartree-Fock approaches, and then we quantify the sensitivity of HIC observables to $L, m_{s}^{*}$ and $m_{v}^{*}$ by analyzing its correlation coefficient by using the transport code, ImQMD-Sky [14], and a strategy to refine the symmetry energy constraints is proposed.

Within the framework of Skyrme-Hartree-Fock approaches, the density dependent of symmetry energy is expressed as,

$$
\begin{aligned}
S(\rho) & =\frac{1}{3} \epsilon_{F} \rho^{2 / 3}+A_{\text {sym }} \rho+B_{\text {sym }} \rho^{\sigma+1}+C_{\text {sym }}\left(m_{s}^{*}, m_{v}^{*}\right) \rho^{5 / 3} \\
& =S_{0}+\frac{L}{3}\left(\frac{\rho-\rho_{0}}{\rho_{0}}\right)+\frac{K_{\text {sym }}}{18}\left(\frac{\rho-\rho_{0}}{\rho_{0}}\right)^{2}+\ldots
\end{aligned}
$$

This formula shows the density dependence of symmetry energy also depends on isoscalar effective mass $\left(m_{s}^{*}\right)$ and isovector effective mass $\left(m_{v}^{*}\right)$ in the mean field level. In Table 1, we list the correlation coefficients $C_{A B}$ [15] between the pair of $K_{0}, S_{0}, L, m_{s}^{*}$ and $m_{v}^{*}$ which are obtained from 120 Skyrme parameter sets. $C_{A B}= \pm 1$ means there is a linear dependence between $A$ and $B$, and $C_{A B}=0$ means no correlations. As shown in Table 1 , the slope of symmetry energy also strongly correlate to $m_{s}^{*}$ and $m_{v}^{*}$ (or effective mass splitting), because the effective mass splitting, $\left(m_{n}^{*}-m_{p}^{*}\right) / m$, is related to $m_{s}^{*}$ and $m_{v}^{*}$ based on the following relationship. Thus, reducing the uncertainties of symmetry energy constraints obtained from heavy ion 
collision data needs us to reduce the uncertainties of constraints on $m_{s}^{*}, m_{v}^{*}$ or $\Delta m^{*} / m$ since all of them are related to the symmetry energy.

$$
\begin{aligned}
\frac{\Delta m *}{m} & =\frac{m_{n}^{*}-m_{p}^{*}}{m}=-\frac{\delta}{m} \frac{2 m_{s}^{*} m_{v}^{*}\left(m_{v}^{*}-m_{s}^{*}\right)}{m_{v}^{* 2}-\delta^{2}\left(m_{v}^{*}-m_{s}^{*}\right)^{2}} \\
& =-2 \frac{m_{s}^{*}}{m}\left[\frac{m_{v}^{*}-m_{s}^{*}}{m_{v}^{*}} \delta+\left(\frac{m_{v}^{*}-m_{s}^{*}}{m_{v}^{*}}\right)^{2} \delta^{2}+\cdots\right.
\end{aligned}
$$

where $\delta=\left(\rho_{n}-\rho_{p}\right) /\left(\rho_{n}+\rho_{p}\right)$.

Table 1: Correlation coefficient $C_{A B}$ between the pair of $K_{0}, S_{0}, L, m_{s}^{*}, m_{v}^{*}$ from 120 Skyrme parameters.

\begin{tabular}{lccccc}
\hline \hline$C_{A B}$ & $K_{0}$ & $S_{0}$ & $L$ & $m_{s}^{*} / m$ & $m_{v}^{*} / m$ \\
\hline$K_{0}$ & 1 & 0.003 & 0.161 & 0.131 & 0.295 \\
$S_{0}$ & 0.003 & 1 & $\mathbf{0 . 7 6 4}$ & 0.397 & 0.228 \\
$L$ & 0.161 & $\mathbf{0 . 7 6 4}$ & 1 & $\mathbf{0 . 4 6 0}$ & 0.212 \\
$m_{s}^{*} / m$ & 0.131 & 0.397 & $\mathbf{0 . 4 6 0}$ & 1 & $\mathbf{0 . 7 1 5}$ \\
$m_{v}^{*} / m$ & 0.295 & 0.228 & 0.212 & $\mathbf{0 . 7 1 5}$ & 1 \\
\hline
\end{tabular}

In order to disentangle the impacts from different $K_{0}, S_{0}, L, m_{s}^{*}$ and $m_{v}^{*}$, we analysis the correlation coefficients $C_{A B}$ between five observables B=CI$R_{2}(n / p)$, CI- $D R(n / p)$, CI- $R_{21}(n / n)$, CI- $R_{21}(p / p)$ and $R_{i}$, and five NM parameters $K_{0}, S_{0}, L, m_{s}^{*}$ and $f_{I}$ in the reaction of ${ }^{124,112} \mathrm{Sn}+{ }^{124,112} \mathrm{Sn}[14,16]$. Here, we use $f_{I}=\frac{1}{2 \delta}\left(\frac{m}{m_{n}^{*}}-\frac{m}{m_{p}^{*}}\right)=\frac{m}{m_{s}^{*}}-\frac{m}{m_{v}^{*}}$ to replace $m_{v}^{*}$ or the effective mass splitting $\Delta m^{*} / m$, because this value is related to the effective mass splitting, and independent of the isospin asymmetry of system and can be easily incorporate into the transport code. All the nucleon yield observables in the simulations are obtained for ${ }^{124} \mathrm{Sn}+{ }^{124} \mathrm{Sn}$ and ${ }^{112} \mathrm{Sn}+{ }^{112} \mathrm{Sn}$ at $E_{\text {beam }}=50$ and $120 \mathrm{MeV} / \mathrm{u}$ at impact parameter $2 \mathrm{fm}$, and for emitted nucleons with $E_{k}>40 M e V$. For isospin diffusion $R_{\text {diff }}$ (or $R_{i}$ ), an additional mixed reaction ${ }^{124} \mathrm{Sn}+{ }^{112} \mathrm{Sn}$ is included and the calculations are performed with mid-peripheral impact parameter of $6 \mathrm{fm}$.

Figure 1 shows the correlation coefficient $C_{A B}$ for $\mathrm{Sn}+\mathrm{Sn}$ reactions at $E_{\text {beam }}=50 \mathrm{MeV} / \mathrm{u}$ (upper panels) and at $E_{\text {beam }}=120 \mathrm{MeV} / \mathrm{u}$ (lower panels) [16]. Red color bars represent positive correlations which mean observable B increases with parameter A, and blue color bars show negative correlations which mean observable B decreases with increasing parameter A. To focus our search for the best experimental observables which are most sensitive to the model parameters, we will discuss only $C_{A B}$ with values greater than 0.6, which were represented as solid blue and red bars in Figure 1. With this 
criterion, $S_{0}$ and $K_{0}$ are not very sensitive to any of the observables studied here.

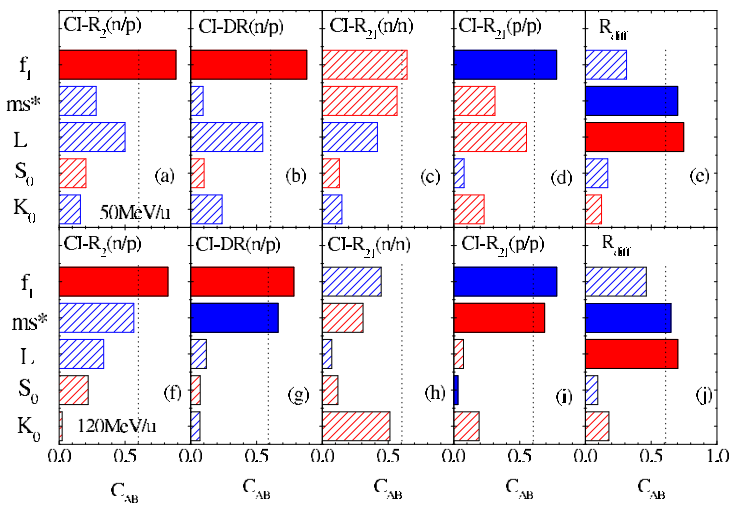

Figure 1: (Color online) Correlations of five obervables, CI- $R_{2}(n / p)$ (a), CI$D R(n / p)$ (b), CI- $R_{21}(n / n)$ (c), CI- $R_{21}(p / p)(\mathrm{d}), R_{\text {diff }}$ (e) with five force parameter, $K_{0}, S_{0}, L, m_{s}^{*}$ and $f_{I}$. Up panels are the results for $50 \mathrm{MeV} / \mathrm{u}$, and bottom panels are for $120 \mathrm{MeV} / \mathrm{u}$.

In the case of L, the slope of the symmetry energy at saturation density, $C_{L, \text { Rdiff }} \sim 0.7$ meets this criterion. $f_{I}$ shows the strongest correlations to the experimental coalescence invariant nucleon observables, the single $\left(R_{2}(n / p)\right)$, double $\mathrm{n} / \mathrm{p}(D R(n / p))$ and isoscaling $\mathrm{p} / \mathrm{p}\left(R_{21}(p / p)\right)$ yield ratios at both incident energies. As shown in panels (a), (f) and (b), (g) of Figure 1, the correlation between the single and double $\mathrm{n} / \mathrm{p}$ yield ratios and $f_{I}$ is larger than 0.8 . Thus, one should be able to extract constraints for both $f_{I}$ and $m_{s}^{*}$ from the single and double $\mathrm{n} / \mathrm{p}$ ratios as well as the $\mathrm{p} / \mathrm{p}$ ratios at $120 \mathrm{MeV} / \mathrm{u}$ incident energy. Alternately, $f_{I}$ can be extracted using the value of $m_{s}^{*} / m=0.65-0.9$ obtained in ref. $[1,5,7,8]$. Our analysis also shows negative correlations of $R_{\text {diff }}$ with $m_{s}^{*}, C_{m_{s}^{*}, R_{\text {diff }}} \sim-0.64$ to -0.70 . The observation that $R_{d i f f}$ is also sensitive to $m_{s}^{*}$ is consistent with the results from BUU calculations $[17,18]$. Strong correlation of single and double $\mathrm{n} / \mathrm{p}$ yield ratios with $m_{s}^{*}$ are also observed mainly at high energy, because the more violent nucleon-nucleon collisions at $120 \mathrm{MeV} / \mathrm{u}$ cause larger momentum transfer and lead to the momentum dependent interaction (which can be characterized by the effective mass) to play more important roles.

In summary, by varying the interaction parameter sets in the ImQMDSky code, we study the influence of different Skyrme parameter sets on the coalescence invariant neutron and proton yield ratios at high energy region, 
for ${ }^{124} \mathrm{Sn}+{ }^{124} \mathrm{Sn}$ and ${ }^{112} \mathrm{Sn}+{ }^{112} \mathrm{Sn}$ at $50 \mathrm{MeV} / \mathrm{u}$ and $120 \mathrm{MeV} / \mathrm{u}$ incident energy. Sensitivities to $S_{0}$ and $K_{0}$ are relatively small, $C_{A B}<0.5$. At incident energy of $120 \mathrm{MeV} / \mathrm{u}$, strong correlations are observed between observables constructed from coalescence invariant nucleon spectra with $E_{c . m .} / A>40$ $\mathrm{MeV}$, such as CI- $R_{2}(n / p)$, CI-DR(n/p), and CI- $R_{21}(p / p)$, and the effective mass splitting as well as the isoscalar effective mass, important input parameters to the transport models. The calculations also confirm the sensitivity of $L$ to the isospin diffusion observable. Since the same observable is also sensitive to isoscalar effective mass, this should allow one to extract the constraints of $m_{s}^{*}$ and $L$ with reasonable uncertainties. Similarly the opposite correlations of the nucleon yield ratios, such as CI-DR(n/p) and CI- $\mathrm{R}_{2}(\mathrm{p} / \mathrm{p})$ ratios to $f_{I}$ and $m_{s}^{*}$, at $120 \mathrm{MeV} / \mathrm{u}$ reactions should allow one to disentangle the effects of the effective nucleon mass splitting and the isoscalar effective mass.

Acknowledgements This work has been supported by the National Key Basic Research Development Program of China under Grant No. 2013CB834404, the National Natural Science Foundation of China under Grants No.(11475262, 11375062,11275072). MBT acknowledges support from the USA National Science Foundation Grants No. PHY-1102511 and travel support from CUSTIPEN (China-US Theory Institute for Physics with Exotic Nuclei) under the US Department of Energy Grant No. DEFG02-13ER42025.

\section{References}

[1] M.Dutra, O. Lourenco, J.S. Sa Martins, and A. Delfino, J.R.Stone, P.D.Stevenson, Phys.Rev.C 85, 035201(2012).

[2] M.Dutra, O. Lourenco, S.S.Avancini, B.V.Carlson, A. Delfino, D.P.Menezes, C.Providencia, S.Typel, J.R.Stone, Phys.Rev.C 90, 055203(2014).

[3] B. A. Brown, Phys. Rev. Lett. 85, 5296 (2000).

[4] B. A. Li, L.W.Chen, C.M.Ko, Phys. Rep 464, 113 (2008).

[5] J. P. Blaizot, J. F. Berger, J. Decharge, and M. Girod, Nucl.Phys. A 591, 435 (1995).

[6] D.H. Youngblood, H.L. Clark, Y.-W. Lui, Phys. Rev. Lett. 82 (1999) 691. 
[7] P. Klupfel, P.-G. Reinhard, T. J. Burvenich, and J. A. Maruhn, Phys.Rev.C79,034310(2009).

[8] Pawel Danielewicz,Nucl.Phys.A673(2000)375.

[9] M. B. Tsang, Yingxun Zhang, P. Danielewicz, M. Famiano, Zhuxia Li, W. G. Lynch and A.W. Steiner, Phys.Rev.Lett. 102, 122701 (2009).

[10] M. B. Tsang, J. R. Stone, F. Camera,et al.,Phys. Rev. C 86, 015803(2012).

[11] J.M.Lattimer, A.W.Steiner, Eur.Phys.J. A50, 40(2014).

[12] C. J. Horowitz, E. F. Brown, Y. Kim, W. G. Lynch, R.Michaels, A. Ono, J. Piekarewicz, M. B. Tsang, and H. H. Wolter, J. Phys. G: Nucl. Part. Phys. 41 (2014) 093001.

[13] D.G. Ireland, W. Nazarewicz, J. Phys. G: Nucl. Part. Phys. 42, 030301(2015).

[14] Yingxun Zhang, M.B.Tsang, Zhuxia Li, Hang Liu, Phys.Lett.B 732, 186(2014).

[15] Siegmund Brandt, Data Analysis-Statistical and Computational Methods for Scientists and Engineers, Fourth Edition, Springer.

[16] Yingxun Zhang, M.B.Tsang, Zhuxia Li, Phys.Lett.B749, 262(2015).

[17] L.W.Chen, Che Ming Ko, Bao-An Li, Phys.Rev.Lett.94, 032701(2005).

[18] J.Rizzo, M.Colonna, V.Baran, M.Di Toro, H.H.Wolter, M.ZielinskaPfabe, Nucl.Phys.A806,79(2008). 